\title{
Porque inserir física moderna e contemporânea no ensino médio? Uma revisão das justificativas dos trabalhos acadêmicos
}

João Ricardo Neves da Silva

Luiz Eduardo Birello Arenghi

Alex Lino

\section{Resumo}

Este trabalho tem por objetivo promover uma revisão bibliográfica das pesquisas acadêmicas que se propõem a investigar a inserção de tópicos de Física Moderna e Contemporânea no Ensino Médio em busca de suas justificativas.Para a realização dessa pesquisa, foram analisados trabalhos acadêmicos dos últimos dez anos da área de Ensino de Ciências que tratam da temática Física Moderna e Contemporânea no Ensino Médio e selecionadas unidades de análise que elencam suas justificativas. As informações foram analisadas por meio da Análise de Conteúdo (BARDIN, 1997) de modo que estas foram categorizadas e comparadas com justificativas apresentadas nos documentos e diretrizes oficiais que versão sobre tal proposta. Conclui-se que as justificativas são bastante próximas das elaboradas por estes documentos, entretanto precisam de uma atualização para que englobem o contexto dos objetivos da educação científica atual.

Palavras-chave: Revisão Bibliográfica, Inserção de Física Moderna e Contemporânea no Ensino Médio, Justificativas.

\begin{abstract}
This work aims to promote a literature review of academic research which propose to investigate the inclusion of topics of Modern and Contemporary Physics in high school in search of their justifications. To carry out this study, we analyzed academic work of the last ten years of the area of researches "Science Education" that worked with the theme Modern and Contemporary Physics in high school and separate units of analysis that we list their justifications. The data were analyzed through content analysis (Bardin, 1997) so they were categorized and compared with reasons given in the documents and official guidelines that version on that proposal. It is concluded that the reasons are quite close to those documents prepared by, however need an update for covering the context of the goals of science education today.
\end{abstract}

Keywords: Literature Review, Inclusion of Modern and Contemporary Physics in High School, Justifications. 


\section{A inserção de física moderna e contemporânea no ensino médio: uma linha de pesquisas em ascensão}

Atualmente, as pesquisas que envolvem o ensino de Física Moderna e Contemporânea (FMC) no Ensino Médio já possuem um número bastante considerável de resultados de forma que esta já possa ser considerada uma linha de pesquisa estabelecida dentro do Ensino de Física. Esta área de pesquisa se desenvolveu principalmente após a década de 1980, no qual os pesquisadores começaram a constatar a desatualização dos currículos escolares em comparação com o progresso da própria Física (TERRAZZAN, 1994). Esse panorama descontextualizado contribui para que os estudantes não conheçam a revolução que ocorreu na Física desenvolvida após o ano de 1900 e, assim, não sejam postos em contato com os parâmetros do desenvolvimento da ciência. Portanto, essa linha de pesquisa se concentra em desenvolver estratégias e metodologias para inserção desses tópicos no ensino de Física.

Sobre o desenvolvimento dessa linha de pesquisa, Sanches (2006) também apresenta um panorama histórico em que desenvolve uma pesquisa sobre a presença dos conteúdos de FMC em sala de aula:

[...] desde a década de 70, a pesquisa na área de ensino de Física vem desenvolvendo parte de sua produção voltada para as novas perspectivas curriculares. [...] Mas, foi somente a partir de meados da década de oitenta que a linha de pesquisa Física Moderna e Contemporânea no Ensino Médio começou a questionar, com maior intensidade, os temas de Física tradicionalmente ensinados nas escolas. (SANCHES, 2006, p. 18).

Conforme pesquisa de revisão bibliográfica realizada por Pereira e Ostermann (2009) em complemento a uma revisão anterior de Ostermann e Moreira (2000),tais autores catalogam as pesquisas nessa linha em quatro tipos principais: 1.) propostas didáticas testadas em sala, 2.) levantamento de concepções, 3.) bibliografia de consulta para professores e 4.) análise curricular.

Ao realizar esta revisão bibliográfica, constatamos que, após cerca de 40 anos de pesquisas nesta vertente, o que mais se encontra na literatura são trabalhos que se dedicam a propor que os conteúdos de FMC sejam transpostos ao ensino numa linguagem acessível a este nível de ensino, como em OSTERMANN (2002), e são encontrados relatos de aplicação de projetos de FMC em amostragens de alunos, visando o estudo de concepções alternativas e identificação de obstáculos para o aprendizado dos novos tópicos. (CABRAL de PAULO, 2006 e LINO, 2010). Em contrapartida, um aspecto essencial em todo o processo de inserção de FMC no EM não tem sido suficientemente investigado, a saber: a preparação - ou formação - dos professores em exercício para a prática desses tópicos em sala de aula.

Os autores também mostram que, do ano 2000 até o ano de 2007, o número de pesquisas nesta linha aumentou consideravelmente, o que a tornauma linha consolidada dentro 
da área. No entanto, ao passo que o número de publicações aumentou de uma revisão para a outra, notamos que os objetivos dessas pesquisas ainda precisam ser reavaliados, uma vez que a maioria delas se dedica à proposição de materiais didáticos para professores, relevando algumas características importantes na pesquisa acadêmica (PEREIRA E OSTERMANN, 2009). Essas categorias nos expressam apenas os tipos de pesquisas que estão sendo feitas nessa linha, mas não se preocupam - mesmo porque este não é o objetivo do trabalho em questão - com outras características desses trabalhos que não seja o seu foco de pesquisa.

Ainda sobre os recentes esforços em promover essa inserção, temos que o conteúdo de FMC foi incluído na estrutura curricular do estado de São Paulo e é desenvolvido nas escolas desde o ano de 2008 como proposta(SÃO PAULO, 2008), passandocomo conteúdo oficial em2010 (SÃO PAULO, 2010), além do fato de que muitos livros didáticos já englobam as proposições referentes à FMC em seus textos (SANCHES, 2006). Sendo assim, há recomendações e propostas curriculares que já versam sobre essa inserção e se torna pertinente entender a forma como a pesquisa acadêmica da área justifica este fato, ou seja, é necessária uma visão mais ampla dos argumentos das pesquisas acadêmicas em defesa da inserção de FMC no Ensino Médio.

Com este panorama apresentado, o que motiva esta pesquisa é uma pergunta razoavelmente simples, mas que suscita uma discussão detalhadasobre as razões pelas quais uma mudança curricular tem sido realizada no ensino de Física no Brasil: Na visão dos pesquisadores, porque é importante inserir Física Moderna e Contemporânea no Ensino Médio?

Essa pergunta é cabível dado o fato de que muitos são os motivos pelos quais seria importante tal inserção e também que muitos trabalhos têm se baseado em razões que não correspondem aos objetivos da educação científica do Ensino Médio no Brasil.

Com isso, este trabalho de revisão bibliográfica tem a pretensão de promover uma revisão das dissertações e teses que se dedicaram ao tema "Física Moderna e Contemporânea no Ensino Médio" no Brasil além de selecionar as justificativas que esses trabalhos apontam para essa inserção e ainda promover uma análise de conteúdo dos argumentos dos pesquisadores para a defesa desta inserção em comparação com os objetivos da educação científica no Brasil.

Nesse sentido, optamos por desenvolver uma análise dos trabalhos acadêmicos - teses e dissertações -principalmente por esses dedicarem uma maior atenção às justificativas e motivações do trabalho quando comparados com artigos científicos de periódicos. 


\section{A seleção do material analisado e a metodologia de análise}

Para a seleção do material analisado, optamos por realizar uma busca no portal de teses e dissertações da CAPES por todos os trabalhos acadêmicos defendidos nos últimos 10 anos no Brasil que contivessem palavras-chave tais como "Física Moderna e Contemporânea", "Inserção de Física Moderna e Contemporânea no Ensino Médio", "Ensino de Física Moderna e Contemporânea", além de uma busca aos sites de todos os programas de pós-graduação da área de "Ensino de Ciências e Matemática" na procura de trabalhos que atendessemà temática. Esses trabalhos foram divididos por ano de defesa e lidos em busca de suas justificativas, motivações e todos os trechos que se relacionassem com aos argumentos do trabalho sobre a importância da introdução de tópicos de FMC no Ensino Médio.As teses e dissertações obtidas foram separadas e enumeradas por ano de defesa. Ao todo foram obtidas 4 teses e 25 dissertações, totalizando 29 trabalhos analisados.

Para a análise dos excertos dos trabalhos selecionados foram utilizadas as premissas metodológicas da Análise de Conteúdo (BARDIN, 1977). A Análise de Conteúdo é um procedimento metodológico deanálise de dados que se coloca em um seguimento mais amplo da teoria da comunicação, além de permitir ao pesquisador fazer inferências sobre os elementos desta comunicação.

Ainda segundo Bardin (1977), a Análise de Conteúdo se caracteriza por ser um conjunto de técnicas de análise das comunicações, que visam obter, por meio de procedimentos sistemáticos e objetivos, indicadores que permitam fazer inferências a partir de conhecimentos relativos às condições de produção e recepção das mensagens veiculadas na comunicação. De um lado, o método pressupõe um trabalho exaustivo de divisões, comparações e aperfeiçoamentos. De outro, pressupõe uma atitude de "vigilância crítica", que implica: "dizer não à ilusão de transparência", "tornar-se desconfiado dos pressupostos", "lutar contra a aparente evidência do saber subjetivo", "destruir a intuição em proveito do construído" e "rejeitar a tentação ingênua de apreender intuitivamente as significações dos protagonistas sociais".

As inferências são de importante finalidade na Análise de Conteúdo. Produzir inferências é interpretar os dados de forma com que seja observado aspectos ocultos, apenas indicados nos processos de comunicação. Bardin (ibid) usa de analogias para exemplificar o processo de inferência: o analista é como um arqueólogo, que trabalha apenas com vestígios, porém estes são manifestações de fenômenos de todos os tipos.

Após os processos de seleção dos documentos e leitura dos registros são formuladas as hipóteses. A hipótese é uma afirmação provisória a qual nos propomos verificar recorrendo aos procedimentos de análise. Esta é uma conjectura que fica suspensa enquanto não é submetida à prova de dados fidedignos. Levantar uma hipótese é perguntar-nos sobre a veracidade da mesma tal como a análise do problema nos sugere. A partir dos objetivos deste trabalho, temos as 
hipóteses de que as justificativas para a inserção de FMC no Ensino Médio têm se repetido a partir dos primeiros trabalhos dessa linha de modo que hoje em dia pouco se enquadram nos objetivos da educação científica proposta.

Os indiciadores são correspondem à frequência em que são observados e analisados os índices em evidência. Os índices podem ser uma menção, explícita ou implícita, de uma mensagem. Nesse sentido, quando esses índices são explicitados passam a ter maior importância para a análise de dados a medida que forem frequentemente mencionados.

Com essas orientações de análise e os objetivos e hipóteses definidos, acreditamos poder contribuir para um avanço na compreensão da importância e principalmente na defesa dos trabalhos futuros que se dedicarem a esta linha de pesquisas.

\section{Porque é importante ensinar física moderna e contemporânea no ensino médio: descrição e interpretação dos dados}

Com as orientações metodológicas apresentadas anteriormente, foi possível reunir em "categorias de análise" todos os trechos que remetem à justificativa dos trabalhos lidos e, em seguida, tecer algumas considerações sobre essas justificativas, por meio de comparações entre elas. $O$ que se mostra neste trabalho são algumas seleções de análise mais representativas de cada categoria e que consideramos exemplificarem como foram analisados os dados, sendo que as quatro categorias aqui apresentadas correspondem ao conjunto das quatro justificativas mais defendidas.Ressaltamos, portanto, que os dados apresentados aqui em forma de unidades de análise (UA) são indicadores de resultado, não sendo transcritas todas as UA para que fosse possível nos atermos às interpretações e inferências sobre os dados.

A seguir, então, discutimos as quatro categorias de justificativas mais encontradas nos trabalhos acadêmicos sobre "Porque é importante inserir Física Moderna e Contemporânea no Ensino Médio?".

\section{Categoria A: A inserção de FMC no EM é importante para a compreensão das tecnologias da atualidade}

As UA elencadas na primeira categoria foram nomeadas de UA-1 até UA-27. Nesta categoria se reúnem os elementos que divulgam a ideia de que a aprendizagem de FMC no Ensino Médio possibilitará aos alunos melhor compreensão a respeito das tecnologias atuais, como podemos destacar nas unidades de análise apresentadas abaixo como exemplo. 
UA-1: Na época atual, Ciência e Tecnologia apresentam-se fortemente associadas, possibilitando a obtenção de aplicações que resultam em maior controle dos fenômenos naturais e permitem gerar benefícios para as pessoas.

UA-18:Com base em seus princípios, surgiram tecnologias cuja importância se destaca no dia-a-dia, tais quais o transistor, essencial nos computadores; o laser, utilizado nas telecomunicações e em tratamentos médicos; e as usinas nucleares, com seus benefícios e riscos associados.

UA-23:É inadmissível que em pleno século XXI, nossos estudantes, no auge do uso das tecnologias promovidas pela Física Moderna, não tenham conhecimento dessa área tão vasta e fascinante da física.

Estes argumentos se encontram na quase totalidade dos trabalhos, principalmente no sentido de manifestar concordância com o trecho expresso nos Parâmetros Curriculares Nacionais (PCN), que afirmam que:

\begin{abstract}
"Alguns aspectos da chamada Física Moderna serão indispensáveis para permitir aos jovens adquirir uma compreensão mais abrangente sobre como se constitui a matéria, de forma que tenham contato com diferentes e novos materiais, cristais líquidos e lasers, presentes nos utensílios tecnológicos, ou com o desenvolvimento da eletrônica, dos circuitos integrados e dos microprocessadores. A compreensão dos modelos para a constituição da matéria deve, ainda, incluir as interações no núcleo dos átomos e os modelos que a ciência hoje propõe para um mundo povoado de partículas. Mas será também indispensável ir mais além, aprendendo a identificar, lidar e reconhecer as radiações e seus diferentes usos". (BRASIL, 2002, P. 210).
\end{abstract}

Assim, temos aqui a categoria de justificativa mais encontrada nos trabalhos analisados, o que pode ter relação com a necessidade de concordar com o argumento expresso claramente no documento oficial de abrangência nacional, destacando assim a importância da pesquisa para a manutenção da ideologia vigente nos documentos públicos.

\title{
Categoria B: A necessidade de atualização curricular do Ensino Médio
}

As justificativas elencadas nesta categoria concordam que a inserção de FMC no Ensino Médio é necessária para apoiar a uma cadeia de argumentos que defendem a atualização curricular no Ensino de Física e, contudo, em sua grande maioria, os autores compreendem a inserção de FMC no Ensino Médio como única forma de atualizar o currículo da educação básica 
para a física. Exemplos de UAs da categoria B são mostradas a seguir. As UA elencadas na categoria B foram nomeadas de UA-28 até UA-43

UA-29:"Em particular, o conjunto de ideias reunidas sob a denominação Física Moderna, desenvolvida desde o final do século XIX até o período contemporâneo - englobando a Teoria da Relatividade, a Mecânica Quântica, a Física Nuclear, a Física de Partículas, a Física da Matéria Condensada e a Cosmologia - quase não é abordado no Ensino Médio."

UA-31:"Embora os Parâmetros Curriculares Nacionais para o Ensino Médio proponham o enriquecimento dos currículos com a incorporação de noções sobre a Física Moderna e a maneira peculiar como se constroem conhecimentos no campo da Ciência, seu efeito tem sido fracamente sentido na maior parte dos estabelecimentos de ensino."

UA-37:"Em suas propostas, os Parâmetros Curriculares Nacionais (PCNs) ressaltam a importância de renovar os currículos escolares."

UA-40:"Estamos no inicio do século XXI e ainda temos a Física Clássica determinística ensinada nas escolas de Ensino Médio (EM) como única e absoluta, fazendo com que o atual currículo de Física fique desatualizado em relação ao avanço científico ocorrido desde o início do século XX até o presente, com o surgimento de novos modelos científicos e novas áreas da ciência."

UA-43:"Desta forma, podemos ainda ter a hipótese de que quando o aluno se depara com um estudo tão antigo da Física, pode ter a impressão de que a ciência não sofreu avanços significativos e sempre será deste mesmo jeito, sem perspectivas de mudanças."

Aqui percebemos uma constante ênfase em fatos como,(a) os elementos teóricos da FMC não são abordados no Ensino Médio, (b) as recomendações dos documentos públicos sobre a aplicação da FMC não refletem na prática em sala de aula, ou (c) os documentos públicos apontam a necessidade de atualização curricular, por isso a necessidade de inserção de FMC no Ensino Médio.

Essas justificativas têm origem em uma preocupação de pesquisa manifestada pelos pioneiros dessa temática, tal como expresso no trecho abaixo

Segundo Alvetti (1999), desde a década de 70, a pesquisa na área de ensino de Física vem desenvolvendo parte de sua produção voltada para as novas perspectivas curriculares. [...] Mas, foi somente a partir de meados da década de oitenta que a linha de pesquisa Física Moderna e Contemporânea no Ensino 
Médio começou a questionar, com maior intensidade, os temas de Física tradicionalmente ensinados nas escolas. (Sanches, 2006, p. 18)

Ou seja, a necessidade de atualização curricular era uma necessidade do Ensino de Física na década de 1970 e se constituía na principal justificativa dos trabalhos pioneiros. Entretanto, atualmente (2012) muitos dos trabalhos acadêmicos ainda persistem em elencar essa como uma das motivações para a inserção de FMC no Ensino Médio, mesmo havendo documentos de proposições curriculares que já englobam estes conteúdos, inclusive de abrangência nacional (BRASIL, 2002; SÃO PAULO, 2008). Assim, neste caso, apontamos que uma das principais justificativas apresentadas nos trabalhos acadêmicos brasileiros que versam sobre a temática pesquisada são ancoradas em justificativas de trabalhos pioneiros e já não se aplicam ao cenário atual do Ensino de Física no país.

\section{Categoria C: A Física Moderna e Contemporânea representou uma mudança de paradigma da Física e essa noção de desenvolvimento das ciências se faz necessária no EM}

Fazem parte desta categorias, poucas unidades de análise que identificam na inserção considerada uma forma de discussão com estudante as questões relacionas ás mudanças de paradigma na Física. As UA elencadas na categoria C foram nomeadas de UA-44 até UA-55 e são exemplos destas as seguintes:

UA-46:"A revolução desencadeada pela Física Moderna atingiu, por exemplo, as concepções de espaço, tempo, massa e energia, o entendimento quanto à estrutura do átomo e a compreensão sobre a própria origem e evolução do Universo."

UA-51:"No entanto, neste ultimo século, a quantidade de inovações e rupturas teóricas tem alcançado um numero muito grande se comparado a outros períodos de sua história."

Aqui encontramos trechos muito presentes e defendidos nos poucos trabalhos em que se encontram, além de sustentar uma concepção de que o estudo do desenvolvimento histórico e epistemológico da Física pode contribuir no processo de aprendizagem dos estudantes.Tais contribuições são verificadas, por exemplo, por testes de aprendizagem que mostram que os conceitos são formados de uma forma mais significativa quando os estudantes estudam FMC em seu desenvolvimento histórico e epistemológico, tal como mostram os trabalhos de Lino (2010), Cabral de Paulo (2006) e Machado (2006). 
Essa justificativa é fundamentação principalmente para os trabalhos que versam sobre história, epistemologia e aprendizagem de conceitos de FMC por alunos do Ensino Médio e são encontradas, primordialmente, em trabalhos acadêmicos que datam a partir do ano de 2005, provavelmente sendo os poucos trabalhos que despontam na "contramão" da exclusividade das justificativas vistas na categoria $B$, levarem em consideração a já realizada atualização de alguns currículos do Ensino Médio para essa vertente.

\section{Categoria 4: A FMC como subsídio à compreensão e crítica das questões atuais que envolvem ciência, tecnologia, sociedade e ambiente}

As UA elencadas na quarta categoria foram nomeadas de UA-56 até UA-76 e com elas podemos inferis que a compreensão dos aspectos relacionados à Ciência e Tecnologia e a possibilidade de crítica deste são, hoje em dia, um parâmetro muito defendido nas pesquisas em Ensino de Ciências. Assim, as unidades de análise presentes nesta categoria contemplam argumentos em defesa dessa abordagem.

UA-58:é essencial que o currículo escolar propicie saberes para os estudantes poderem acompanhar criticamente os desdobramentos dessas inter-relações mesmo após a conclusão de sua educação formal.

UA-62:Porém, paradoxalmente, a inércia verificada na renovação de currículos e práticas pedagógicas pode acabar deixando os alunos à margem da cultura científica e tecnológica do mundo moderno, devido à defasagem existente entre o que é aprendido na escola e os fatos em andamento na Sociedade.

UA-68:quando se incluem assuntos que contribuem para o entendimento de questões atuais e relevantes.

UA-74:um ensino que traga para a sala de aula ideias atuais e capazes de contribuir para a formação abrangente do estudante, permitindo-lhe compreender princípios básicos da Ciência e habilitando-o a participar de debates envolvendo questões científicas e tecnológicas que repercutam na sociedade e no ambiente.

O conjunto de unidades de análise que compõe esta categoria se pautam no já relatados trabalhos e se relacionam a vertente de pesquisa sobre relação entre Ciência, Tecnologia e Sociedade, que está em grande ascensão no país (FRANCO-DOS-SANTOS et al, 2011). Esta ideia de que o estudo das teorias contemporâneas proporciona aos alunos a oportunidade de participação em decisões e debates que envolvem o uso da ciência na sociedade (Lopes, 2010),(Carvalho, 
2005),(Reis, 1999) tem se tornado uma grande empreitada no Ensino de Ciências no Brasil. Há, inclusive, a relação que também se pode fazer com o documento oficial de abrangência nacional, que engloba os mesmos princípios formativos ao tratar o ensino de FMC.

... a física deve apresentar-se como um conjunto de competências específicas que permita perceber como lidar com os fenômenos naturais e tecnologias, presentes no cotidiano, de forma contextualizada, em articulação com competências de outras áreas, de forma a mostrar que o conhecimento proporcionado por ela possa se transformar em uma ferramenta nas formas de pensar e agir. (Brasil, 2002, p. 37).

Assim, segundo esse conjunto de trabalhos e justificativas, o estudo de FMC proporcionaria conteúdo para a compreensão do desenvolvimento da ciência e da tecnologia e a desatualização do currículo deixaria os estudantes sem subsídios para acompanharem os desenvolvimentos tecnológicos da atualidade e poderem se posicionar perante as consequências de tal avanço.

A categoria E, clamada de "outras", mostra algumas poucas UA com justificativas aleatórias que não fora englobadas nas quatro principais categorias e que estão contidas em trabalhos que também apresentam as quatro outras justificativas como principais. Um exemplo de UA da categoria E é uma afirmação de que o conhecimento de Física Moderna e Contemporânea representa uma possibilidade de o aluno conviver com o uso de ferramentas matemáticas mais avançadas com o apoio do fenômeno físico para sua compreensão.

Uma sistematização geral de todas as UA encontradas e as categorias que abrangem é mostrada na tabela a seguir, para fins de compreensão geral da análise de conteúdo realizada.

\begin{tabular}{|c|c|}
\hline UAs & Categoria \\
\hline UA-1 até UA-27 & $\begin{array}{c}\text { Categoria A: A inserção de FMC no EM é } \\
\text { importante para a compreensão das } \\
\text { tecnologias da atualidade }\end{array}$ \\
\hline UA-28 até UA-43 & $\begin{array}{c}\text { Categoria B: A necessidade de atualização } \\
\text { curricular do Ensino Médio }\end{array}$ \\
\hline UA-44 até UA-55 & $\begin{array}{c}\text { Categoria C: A Física Moderna e } \\
\text { Contemporânea representou uma mudança de } \\
\text { paradigma da Física e essa noção de } \\
\text { desenvolvimento das ciências se faz necessária } \\
\text { no EM. }\end{array}$ \\
\hline
\end{tabular}




\begin{tabular}{|c|c|}
\hline UA-56 até UA-76 & $\begin{array}{c}\text { Categoria D: A FMC como subsídio à } \\
\text { compreensão e crítica das questões atuais que } \\
\text { envolvem ciência, tecnologia, sociedade e } \\
\text { ambiente. }\end{array}$ \\
\hline UA-76 até UA-83 & Categoria E: Outras \\
\hline
\end{tabular}

Tabela 2: Sistematização das UAs pertencentes à cada categoria.

\section{Discussão dos resultados analisados}

O que elencamos aqui são exemplos de unidades de análise que compõe as quatro principais categorias de justificativas encontradas nos trabalhos acadêmicos para a inserção de FMC no Ensino Médio. A seguir, apresenta-se uma tabela com os números de cada categoria de justificativas no geral dos trabalhos analisados. Como os trabalhos apresentam, na sua grande maioria, muitas justificativas e todas foram consideradas, a tabela engloba um total geral de mais de $100 \%$, pois há muitos trabalhos que apresentam justificativas pertencentes à mais de uma categoria. Lembrando que, no total, foram analisados 29 trabalhos.

\begin{tabular}{|c|c|}
\hline \multicolumn{2}{|c|}{ Numero de trabalhos com justificativas em cada categoria } \\
\hline Categoria & Quantidade \\
\hline Total de trabalhos analisados & 29 \\
\hline Categoria A & 27 \\
\hline Categoria B & 16 \\
\hline Categoria C & 12 \\
\hline Categoria D & 21 \\
\hline Outras & 7 \\
\hline
\end{tabular}

Tabela 2: Número de trabalhos em cada categoria de justificativas

Dessa maneira, temos aqui representados os principais dados de uma revisão bibliográfica acerca de como os autores de pesquisas realizadas em âmbito acadêmico têm justificado a necessidade de inserção de tópicos de FMC a na estrutura curricular do Ensino Médio brasileiro.

Com isso, podemos resumir as principais justificativas encontradas em quatro grandes grupos: (A) compreensão dos aparatos tecnológicos modernos; (B)atualização curricular dos R. B. E. C. T., vol 6, núm. 1, jan-abr.2013 ISSN - 1982-873X 
conteúdos de física do ensino médio; (C) necessidade de compreensão das revoluções científicas desencadeada pelas proposições referentes à FMC e (D) necessidade de uma formação voltada para o debate de questões científicas atuais.

\section{Conclusões}

Esta revisão bibliográfica pretendeu discutir, a partir de uma análise dos trabalhos acadêmicos publicados no Brasil nos últimos dez anos, o que os pesquisadores em Ensino de Física têm defendido quando se trata de justificar a importância do trabalho com os conteúdos pertencentes à FMC no Ensino Médio.

A primeira discussão que se torna interessante neste ponto é a de que a justificativa mais encontrada tem raízes em um contexto no qual não haviam pesquisas relacionadas à essa inserção e vêm sendo propagadas até os dias atuais como se o cenário não tivesse mudado,mostrando que os pesquisadores estão buscando atender - ou cumprir - as recomendações oficiais governamentais, mesmo que muitas delas ainda digam que estão longe de conseguirem na prática o que esses documentos promulgam.

Nota-se que as quatro principais justificativas encontradas são expressões de ideias já presentes nos documentos oficiais, tais como a necessidade de atualização curricular no ensino de física no ensino médio ou a noção de que o desenvolvimento tecnológico da atualidade pode ser compreendido mediante aprendizagem desses conteúdos de FMC. Contudo, também apontamos o fato de que muitas das argumentações presentes nos trabalhos fazem parte de um processo de reprodução das determinações dos documentos oficiais e também de uma "rede" de reprodução das justificativas dos trabalhos pioneiros da área e que não correspondem mais às necessidades da área no cenário atual, como as apontadas na categoria B. As escolas brasileiras atuais já englobam em seus documentos a necessidade de tal inserção, de modo que as pesquisas acadêmicas mais atuais deveriam concentrar suas motivações e seus esforços de argumentação na defesa de outros "porquês".

Outras justificativas se apoiam em tendências de pesquisa teóricas que marcam as pesquisas em Ensino de Física, como a necessidade da compreensão da epistemologia da Ciência e o fato de a FMC ter representado uma mudança de paradigma para a Física além da questão da formação para a participação no coletivo e das decisões que envolvem a Ciência. Na compreensão desses autores, as justificativas apontadas nas categorias C e D representam melhor as necessidades da educação em ciências da atualidade com respeito à inserção de FMC no Ensino Médio, pois, uma vez que os currículos se encontram, em sua maioria, atualizados, busca-se atualmente por uma compreensão do desenvolvimento dessa física, de seu caráter puramente paradigmático e do uso do conhecimento moderno como meio de formação de jovens participantes nas decisões que envolvem ciência e tecnologia. 
Com este trabalho esperamos poder contribuir com futuras pesquisas nesta linha pesquisa no sentido de destacar como a comunidade acadêmica observa essa necessidade de atualização curricular e deixar as sementes para proposições que beirem a inovação curricular no Ensino de Física, uma vez que temos a convicção de que há ainda muitas outras justificativas e muitos outros motivos para o investimento de esforços no estudo da formação científica no Brasil.

\section{Referências}

BARDIN, L. (1977). Análise de Conteúdo. Tradução de Luís Antero Reto e Augusto Pinheiro Lisboa: Edições 70.

BRASIL. (2002). Ministério da Educação. PCN+ Ensino Médio: Orientações Complementares aos Parâmetros Curriculares Nacionais para o Ensino Médio. Ciências da Natureza, Matemática e suas tecnologias. Brasília.

FRANCO-DOS-SANTOS, P. G; LOPES, N. C; CARNIO, M. P; BIRELLO ARENGHI, L. E. PEDRANCINI, V. D. CARVALHO, W. L. P. ORQUIZA DE CARVALHO, L. M. A abordagem de questões sociocientíficas no ensino de ciências: uma compreensão das sequências didáticas propostas por pesquisas na área. Em: Anais da XII Reunião Técnica e II Encontro dos Grupos de Pesquisa, Bauru - SP, 2011.

LINO, A. (2010). Inserção de Física Moderna e Contemporânea no Ensino Médio: a ligação entre teorias clássicas e modernas sob a perspectiva da aprendizagem significativa. 2010. Dissertação (Mestrado em Educação para a Ciência e a Matemática). Universidade Estadual de Maringá. Maringá.

MACHADO, D. I. Construção de conceitos de física moderna e sobre a natureza da ciência com o suporte da hipermídia. 2006. 300 f. Tese (Doutorado em Educação para a Ciência). Faculdade de Ciências, UNESP, Bauru, 2006.

OSTERMANN, F. (1999). Tópicos de Física Contemporânea em escolas de nível médio e na formação de professores de Física. 175 f. Tese. Instituto de Física - Universidade Federal do Rio Grande do Sul, Porto Alegre, Rio Grande do Sul.

OSTERMANN, F. MOREIRA, M. A. (2000). Uma revisão bibliográfica sobre a área de pesquisa "Física Moderna e Contemporânea no Ensino Médio". Revista Investigação em Ensino de Ciências do Instituto de Física da Universidade Federal do Rio Grande do Sul, Porto Alegre, v. 05, n. 01. 
PAULO, I. J. C. (2006). A aprendizagem significativa crítica de conceitos de mecânica quântica segundo a interpretação de Copenhagen e o problema da diversidade de propostas de inserção de Física moderna e Contemporânea no Ensino Médio. 235 f. tese (Doutorado em Educação) Departamento de Didácticas Específicas - Universidade de Burgos, Burgos, Espanha.

PAULO, I.J.C. (1997). Elementos para uma proposta de inserção de tópicos de Física Moderna no Ensino de Nível Médio,91 f. dissertação (Mestrado em Educação) - Instituto de Educação Universidade Federal do Mato Grosso do Sul, Cuiabá, Mato Grosso do Sul.

PEREIRA, A. P.; OSTERMANN, F. (2009). Sobre o ensino de Física Moderna e Contemporânea: uma revisão da produção acadêmica recente. Investigações em ensino de Ciências, v. 14, n 3. pp- 393 420.

REIS, P.R. A discussão de assuntos controversos no ensino de ciências. Inovação, vol.12 1999. 106 p.

SANCHES, M. B.(2006). AFísica Moderna e Contemporânea no Ensino Médio: Qual sua presença em sala de aula?108 f. Dissertação (Mestrado em Educação para a Ciência e a Matemática) Universidade Estadual de Maringá, Maringá, Paraná.

SÃO PAULO. (2008). Secretaria da Educação. Secretaria do Estado de São Paulo; Proposta Curricular do Estado de São Paulo: Física, ensino médio; São Paulo.

SÃO PAULO.(2009). Secretaria da Educação. Secretaria do Estado de São Paulo; Caderno do professor: física, ensino médio - 3ạ 'serie. v.3; São Paulo.

SÃO PAULO. (2010). Secretaria da Educação. Secretaria do Estado de São Paulo. Currículo do Estado de São Paulo: Ciências da Natureza e suas tecnologias; São Paulo.

João Ricardo Neves da Silva- Mestre em Educação para a Ciência e a Matemática pela Universidade Estadual de Maringá (UEM) e Doutorando em Educação para a Ciência pela Faculdade de Ciências da UNESP - Campus de Bauru - jicicardo.fisica@gmail.com

Luiz Eduardo BirelloArenghi- Mestre e Doutorando em Educação para a Ciência pela Faculdade de Ciências da UNESP - Campus de Bauru - luisbirello@yahoo.com.br 
Alex Lino - Mestre e Doutorando em Educação para a Ciência e a Matemática pela Universidade Estadual de Maringá (UEM) - alexlinop@hotmail.com 